\title{
Influência da composição de catalisadores de Pt e Ru na reação de eletro-oxidação de propanodiol
}

\author{
Edervaldo Buffon ${ }^{1,2}(\mathrm{IC})$; Maria de Lourdes S. Vasconcellos ${ }^{1 *}(\mathrm{IC})$; Paulo Olivi ${ }^{3}$ (PQ); \\ Demetrius Profeti ${ }^{1}(\mathrm{PQ})$; Luciene P.R. Profeti ${ }^{1}(\mathrm{PQ})$. \\ ${ }^{1}$ Departamento de Química e Física, Universidade Federal do Espírito Santo, Alegre, ES, Brasil. \\ ${ }^{2}$ Departamento de Química Analítica, Instituto de Química, Universidade Estadual Paulista, Araraquara, SP, Brasil. \\ ${ }^{3}$ Departamento de Química, Universidade de São Paulo, Ribeirão Preto, SP, Brasil \\ *e-mail: lourdes-vasconcellos@hotmail.com
}

\section{Resumo}

Neste trabalho foram preparados electrocatalisadores contendo platina e óxido de rutênio em diferentes proporções. A atividade catalítica destes materiais foi avaliada durante a oxidação de propanodiol. Este álcool é obtida de forma renovável através da hidrogenólise do glicerol, o qual é um subproduto da produção de biodiesel. Desta forma, esta molécula representa uma possibilidade para a substituição do hidrogênio utilizado em células a combustível, uma vez que $\mathrm{o}$ uso do hidrogênio nesses dispositivos é desvantajoso devido à dificuldade de armazenamento e risco de explosão. A atividade catalítica dos eletrodos contendo Pt e Ru foi investigada através das técnicas de voltametria cíclica e cronoamperometria e os resultados obtidos demostraram uma atividade catalítica superior do eletrodo composto por $60 \% \mathrm{Pt}$ e $40 \%$ $\mathrm{Ru}$, indicando a potencial aplicação deste eletrodo na catálise da reação de eletro-oxidação desta molécula orgânica.

\begin{abstract}
In this work were prepared electrodes coated with films containing platinum and ruthenium oxide in different proportions. The catalytic activity of these devices was evaluated during the oxidation of propanediol molecule, which is obtained from renewable sources by hydrogenolysis of glycerol which is a byproduct of biodiesel production. Thus, this molecule is a possibility for the replacement of hydrogen used in fuel cells, since the use of hydrogen in these devices is disadvantageousharmful due to difficulty in storage and risk of explosion. The catalytic activity of the electrodes containing $\mathrm{Pt}$ and $\mathrm{Ru}$ was investigated through the techniques of cyclic voltammetry and chronoamperometry and the results showed a higher catalytic activity of electrode composed of $60 \% \mathrm{Pt}$ and $40 \% \mathrm{Ru}$, indicating the potential application of this electrode at catalysis of the electro-oxidation reaction of that organic molecule.
\end{abstract}

Keywords (Palavras chaves): Eletro-oxidação de Álcoois, Propanodiol, Platina e Rutênio.

\section{Introdução}

Atualmente, há um grande interesse na produção de catalisadores que atuem com eficiência na eletro-oxidação de moléculas orgânicas, como por exemplo, etanol e glicerol. Tais substâncias podem ser utilizadas para substituir o hidrogênio gasoso utilizado como combustível em células a combustível. O uso do hidrogênio gasoso nesses dispositivos é desvantajoso por sua dificuldade de armazenamento e risco de explosão [1].
Dentre os catalisadores já investigados, os metais nobres, como platina, apresentam maior eficiência na catálise da oxidação de moléculas orgânicas. Contudo, estes materiais sofrem uma perda progressiva da atividade catalítica devido à forte adsorção de espécies intermediárias da reação, como o $\mathrm{CO}$, na superfície do eletrodo. Para minimizar este efeito, a Pt pode ser combinada com alguns metais que promovam a formação de espécies oxigenadas na superfície. Estas espécies oxigenadas auxiliam na oxidação do $\mathrm{CO}_{\text {ads }} \mathrm{a}$ $\mathrm{CO}_{2}$, em potenciais inferiores ao da platina, impedindo 
assim o envenenamento do catalisador e preservando sua atividade catalítica [2].

Com o objetivo de desenvolver materiais de eletrodos capazes de oxidar álcoois com eficiência, no presente trabalho foram preparados eletrodos recobertos com filmes contendo platina e óxido de rutênio em diferentes proporções sobre suporte de titânio pelo método de Pechini [referencia]. Os eletrodos obtidos foram aplicados na eletrooxidação do propanodiol $\left(\mathrm{C}_{3} \mathrm{H}_{8} \mathrm{O}_{2}\right)$. Este álcool possui características interessantes para ser utilizado como combustível em células a combustível, principalmente pela vantagem de ser produzido de forma renovável através do glicerol, o qual é produzido em grande quantidade durante a produção de biodiesel.

A atividade catalítica dos eletrodos contendo platina e óxido de rutênio durante a reação de eletro-oxidação do propanodiol foi avaliada através das técnicas de voltametria cíclica e cronoamperometria.

\section{Materiais e Métodos}

A preparação dos eletrodos foi baseada no recobrimento de placas de $\mathrm{Ti}\left(2 \mathrm{~cm}^{2}\right)$ por filmes finos constituídos por óxidos de platina e rutênio em diferentes proporções pelo método de Pechini. Neste método os filmes de $\mathrm{Pt}$ e $\mathrm{Ru}$ foram preparados por pincelamento das soluções precursoras contendo os metais de interesse diretamente sobre um substrato de $\mathrm{Ti}$ e depois calcinados em $500{ }^{\circ} \mathrm{C}$ [3]. O método de Pechini foi escolhido para a preparação dos eletrodos por permitir a obtenção de filmes uniformes, superfícies homogêneas e por ser um método químico, permitindo assim, o controle da estequiométrica do óxido formado.

As soluções precursoras de $\mathrm{Pt}$ e $\mathrm{Ru}$ foram preparadas pela dissolução de ácido cítrico em etilenoglicol sob agitação constante e temperatura de $60^{\circ} \mathrm{C}$. Após a completa dissolução do ácido a temperatura foi elevada a 90-95 $\stackrel{\circ}{ } \mathrm{C}$ para que as soluções de $\mathrm{H}_{2} \mathrm{PtCl}_{6}$ ou de $\mathrm{RuCl}_{3}$ pudessem ser adicionadas. A proporção para a preparação destas soluções percussoras foi 1:4:16 em mol de metal ( $\mathrm{Pt}$ ou $\mathrm{Ru}$ ), ácido cítrico e etilenoglicol, respectivamente. Estas soluções foram misturadas na proporção adequada para preparar a camada de óxido na composição desejada e pinceladas sobre a superfície do Ti.

Devido à simplicidade do método, foram preparados eletrodos recobertos por óxidos de $\mathrm{Pt}$ e $\mathrm{Ru}$ em diferentes composições contendo 0, 30, 50, 60, 70, 80, 90 e $100 \%$ de Pt.

Os experimentos eletroquímicos foram realizados em meia célula, utilizando um eletrodo auxiliar de platina e um eletrodo reversível de hidrogênio como referência, e com o auxílio de um potenciostato/galvanostato AUTOLAB PGSTAT $128 \mathrm{~N}$, operado pelo software GPES 4.9.

A técnica de voltametria cíclica foi utilizada para caracterização dos processos redox dos filmes de óxidos e para determinar a atividade catalítica para a eletrooxidação de propanodiol. A técnica de cronoamperometria também foi utilizada para realizar a avaliação eletrocatalítica. O eletrólito suporte utilizado em todos os experimentos foi uma solução de $\mathrm{NaOH}$ na concentração de 1,0 mol.L-1 , uma vez que, em meio básico a velocidade de desprotonação das moléculas orgânicas adsorvidas é superior quando comparada ao meio ácido.

\section{Resultados e Discussões}

Após a preparação dos eletrodos contendo óxidos de platina e rutênio em diferentes composições, realizou-se a caracterização destes materiais pela técnica de voltametria cíclica à velocidade de $10 \mathrm{mV} \mathrm{s}^{-1}$. Os voltamogramas cíclicos foram obtidos em

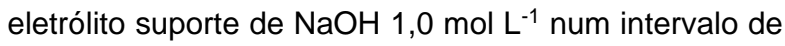
potencial de 0,05 à $1,2 \mathrm{~V}$ versus $\mathrm{ERH}$. Antes de realizar a etapa de caracterização voltamétrica, com a finalidade de hidratar os sítios ativos dos eletrodos, submeteu-se os mesmos a 10 ciclos de varredura de potencial a uma velocidade de $100 \mathrm{mV} \mathrm{s}^{-1}$. Alguns dos resultados obtidos durante a etapa de caracterização voltamétrica são apresentados pelas Figuras 1 e 2:

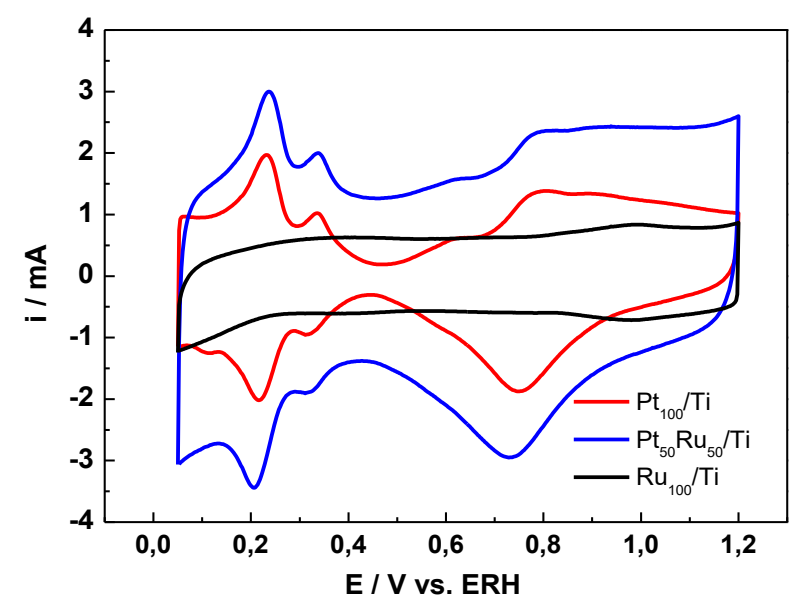

Figura 1: Voltamogramas cíclicos obtidos para os eletrodos de $\mathrm{Pt}_{100} / \mathrm{Ti}$, $\mathrm{Pt}_{50} \mathrm{Ru}_{50} / \mathrm{Ti}$ e $\mathrm{Ru}_{100} / \mathrm{Ti}$ em eletrólito suporte de $\mathrm{NaOH}$ 1,0 $\mathrm{mol} \mathrm{L}^{-1}$ numa velocidade de varredura de $10 \mathrm{mV} \mathrm{s}^{-1}$. 


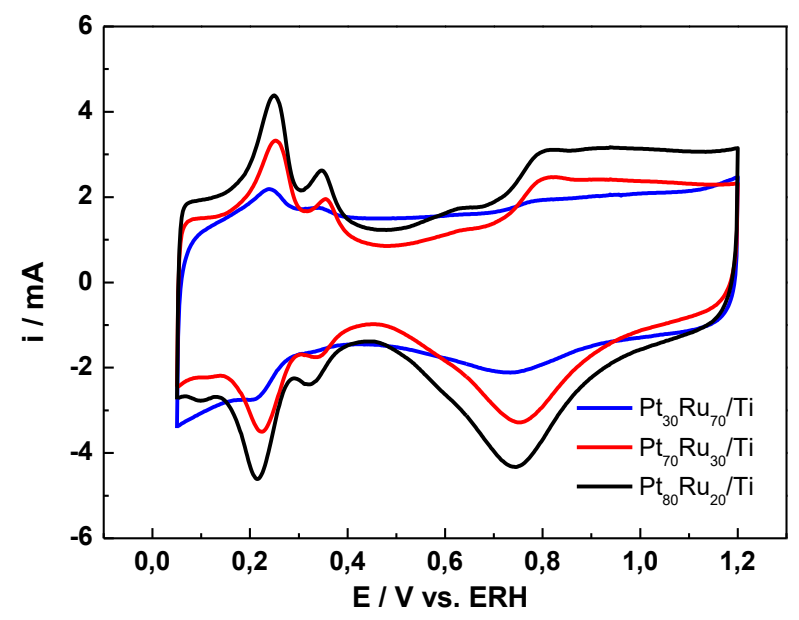

Figura 2: Voltamogramas cíclicos obtidos para os eletrodos de $\mathrm{Pt}_{30} \mathrm{Ru}_{70} / \mathrm{Ti}, \mathrm{Pt}_{70} \mathrm{Ru}_{30} / \mathrm{Ti}$ e $\mathrm{Pt}_{80} \mathrm{Ru}_{20} / \mathrm{Ti}$ em eletrólito suporte de $\mathrm{NaOH}$ $1,0 \mathrm{~mol} \mathrm{~L}^{-1}$ numa velocidade de varredura de $10 \mathrm{mV} \mathrm{s}^{-1}$.

Ao observar na Figura 1 o perfil voltamétrico obtido para o eletrodo de $\mathrm{Pt}_{100} / \mathrm{Ti}$, nota-se um perfil ciclovoltamétrico semelhante à platina policristalina em meio básico: no intervalo de $0,05 \mathrm{~V}$ a $0,4 \mathrm{~V}(\mathrm{ERH})$ aparecem dois picos de oxidação que correspondem à reação de dessorção de hidrogênio. O intervalo de $0,4 \vee$ a $0,8 \vee(E R H)$ corresponde à região de carregamento da dupla camada. A região de $0,8 \mathrm{~V}$ a $1,2 \mathrm{~V}$ na varredura anódica inicia-se o processo de formação dos óxidos de platina $\left(\mathrm{PtOH}, \mathrm{Pt}(\mathrm{OH})_{2}\right.$, $\mathrm{PtO}$, $\mathrm{PtO}_{2}$ ). Na varredura catódica entre $1,2 \mathrm{~V}$ e 0,4 $\mathrm{V}(\mathrm{ERH})$ há um pico em aproximadamente $0,8 \vee(E R H)$ correspondente à redução de todas as espécies de óxidos de Pt formados, e de $0,4 \mathrm{~V}$ a $0,0 \vee(E R H)$ observamos dois picos de redução referentes ao processo de adsorção de hidrogênio na superfície do eletrodo.

Analisando na Figura 1 o voltamograma cíclico obtido para o eletrodo de $\mathrm{Ru}_{100} / \mathrm{Ti}$, observa-se um par de pico reversível em aproximadamente $1,0 \mathrm{~V}$ característico do processo redox desta espécie metálica. Já no voltamograma cíclico obtido para o eletrodo contendo $50 \%$ Pt e 50\% Ru observa-se a existência dos processos redox característicos da platina policristalina em meio básico e do processo redox referente ao rutênio, indicando um comportamento misto do eletrodo binário. Além disso, observa-se também o aumento da carga voltamétrica do eletrodo binário quando comparado ao eletrodo de $\mathrm{Pt}_{100} / \mathrm{Ti}$. De acordo com Laourni et al. [4], este aumento de carga voltamétrica observado para o eletrodo de $\mathrm{Pt}_{50} \mathrm{Ru}_{50} / \mathrm{Ti}$ pode ser atribuído a combinação dos dois elementos metálicos, o que provoca um aumento na quantidade de poros e trincas na superfície do eletrodo, influenciando diretamente na atividade catalítica deste material.

A Figura 2 apresenta os perfis voltamétricos obtidos para os eletrodos contendo 30,70 e $80 \%$ de $\mathrm{Pt}$, onde observa-se o aumento da carga voltamétrica referente aos picos de adsorção e de dessorção de hidrogênio à medida que ocorre o aumento da quantidade de Pt na composição do eletrodo.

De acordo com Gomes et al. [5], a eletrooxidação do propanodiol (propano-1,2-diol) em eletrodos contendo platina envolve a adsorção química da espécie orgânica na superfície do eletrodo de trabalho através da desidrogenação do carbono-alfa. Os produtos da reação de oxidação do propanodiol são Lactaldeido e Monohidroxiacetona [6], conforme pode ser observado pela Figura 3:

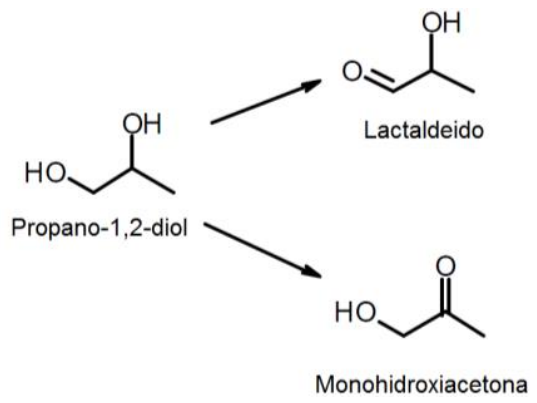

Figura 3: Produtos da reação de oxidação da molécula de propano-1,2-diol.

O voltamograma cíclico obtido durante a eletrooxidação de uma solução de propanodiol $1,0 \mathrm{~mol} \mathrm{~L}^{-1} \mathrm{em}$ meio básico utilizando o eletrodo contendo $70 \%$ Pt e $30 \%$ Ru é apresentado pela Figura 4:

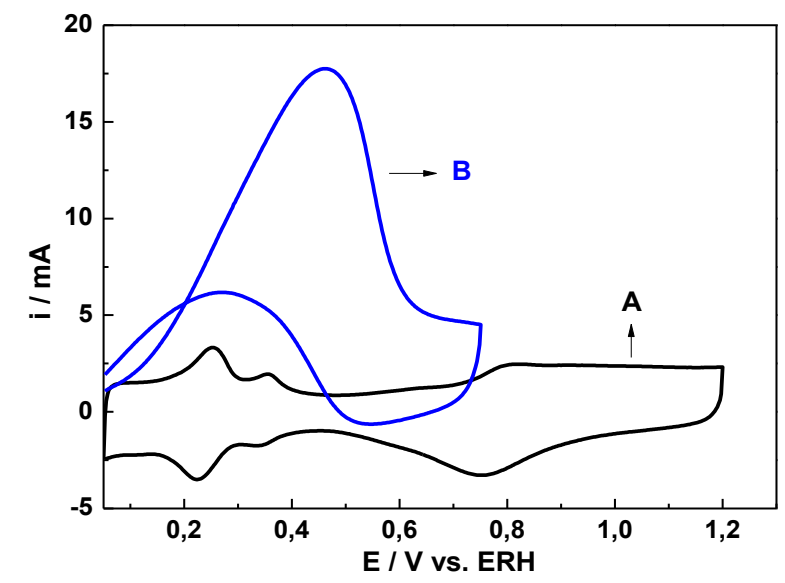

Figura 4: Voltamogramas cíclicos obtidos para o eletrodo $\mathrm{Pt}_{70} \mathrm{Ru}_{30} / \mathrm{Ti}$

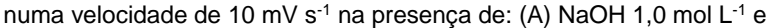
(B) Propanodiol 1,0 mol L-1 em NaOH 1,0 $\mathrm{mol} \mathrm{L}^{-1}$.

Ao analisar o voltamograma cíclico para o eletrodo $\mathrm{Pt}_{70} \mathrm{Ru}_{30} / \mathrm{Ti}$ na presença de propanodiol $1,0 \mathrm{~mol} \mathrm{~L}^{-1}$, observa-se que a oxidação da molécula orgânica provoca um aumento nos valores da corrente de pico anódica atingindo um potencial de pico de aproximadamente $500 \mathrm{mV}$. Esse comportamento ciclo voltamétrico está de acordo com a literatura de Maltanava et al. [7] para a oxidação da molécula de propanodiol. 
Avaliou-se a atividade catalítica dos eletrodos contendo platina e rutênio na oxidação do propanodiol através das técnicas de voltametria cíclica e cronoamperometria. A Figura 5 compara a varredura anódica dos voltamogramas cíclicos dos eletrodos obtidos em solução de eletrólito suporte de $\mathrm{NaOH} 1,0$ mol L-1 na presença de propanodiol $1,0 \mathrm{~mol} \mathrm{~L}^{-1}$.

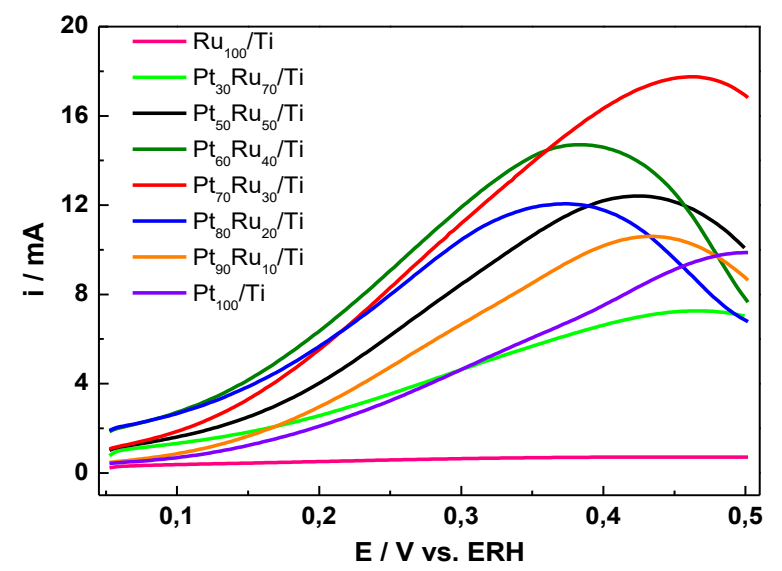

Figura 5: Varredura anódica dos voltamogramas cíclicos dos eletrodos obtidos para a oxidação de Propanodiol $1,0 \mathrm{~mol} \mathrm{~L}^{-1} \mathrm{em} \mathrm{NaOH}$ $1,0 \mathrm{~mol} \mathrm{~L}^{-1}$ numa velocidade de $10 \mathrm{mV} \mathrm{s}^{-1}$.

A Figura 5 apresenta o comportamento voltamétrico de todos os eletrodos utilizados durante a eletro-oxidação do propanodiol, onde nota-se que os eletrodos contendo platina em sua composição apresentaram um pico anódico numa faixa de potencial entre 370 e 460 $\mathrm{mV}$ referente a oxidação da molécula orgânica. A variação nos valores de potencial de pico anódico pode ser atribuída as diferentes composições dos eletrodos.

Observa-se ainda ao analisar a Figura 5 que todos os eletrodos binários apresentaram uma atividade catalítica superior ao eletrodo $\mathrm{Pt}_{100} / \mathrm{Ti}$ em potenciais inferiores a $300 \mathrm{mV}$, indicando que a adição de rutênio contribuiu para o aumento na atividade catalítica. Além disso, percebe-se a ausência de atividade catalítica para o eletrodo $\mathrm{Ru}_{100} / \mathrm{Ti}$.

Realizou-se a eletrooxidação da molécula de propanodiol utilizando a técnica de cronoamperometria com objetivo de avaliar a eficiência dos eletrodos numa condição estacionária, a qual mais se aproxima da condição de operação de uma célula a combustível. Nessa técnica, aplica-se um valor de potencial fixo e monitora-se a corrente obtida por um tempo definido. Para análise cronoamperométrica dos eletrodos contendo Pt e Ru em meio básico durante a oxidação do propanodiol, aplicou-se o potencial fixo de $300 \mathrm{mV}$ $(\mathrm{ERH})$ durante 10 minutos. $\mathrm{O}$ perfil cronoamperométrico obtido para os eletrodos utilizados é apresentado pela Figura 6:

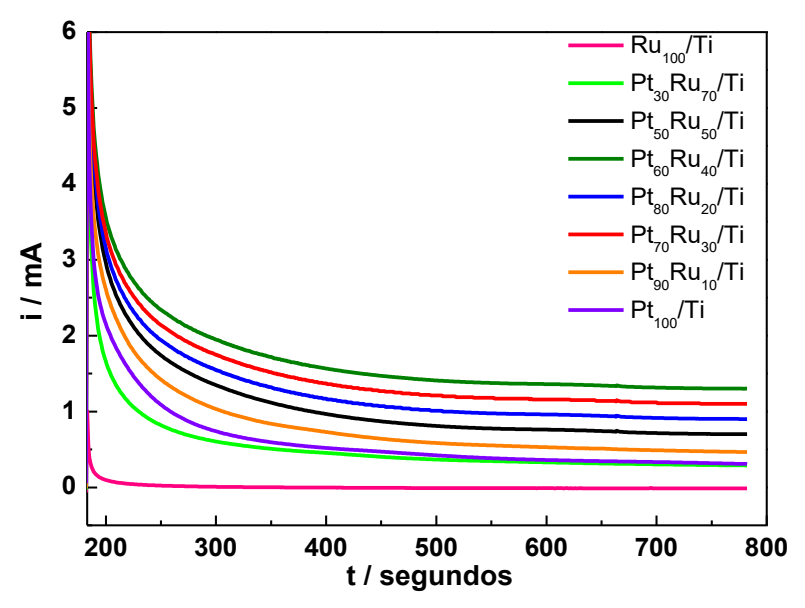

Figura 6: Cronoamperograma de oxidação de $1,0 \mathrm{~mol} \mathrm{L^{-1 }}$ de Propanodiol em 1,0 mol L-1 de $\mathrm{NaOH}$, em potencial fixo de $300 \mathrm{mV}$ para os eletrodos contendo Pt e Ru em diferentes proporções.

Ao observar a Figura 6, nota-se que com exceção do eletrodo $\mathrm{Ru}_{100} / \mathrm{Ti}$, todos os outros eletrodos apresentaram valores de corrente superior a zero, indicando atividade para a oxidação de propanodiol. Observa-se ainda uma queda exponencial nos valores de corrente nos primeiros segundos que se mantém constante após aproximadamente 200 segundos até 0 final do experimento. A queda nos valores de corrente ocorre devido ao bloqueio da superfície do eletrodo por fragmentos da molécula orgânica adsorvidos proveniente do processo de oxidação.

Percebe-se que na faixa de potencial investigada de $300 \mathrm{mV}$ a queda nos valores de densidade de corrente é menos acentuada para o eletrodo $\mathrm{Pt}_{60} \mathrm{Ru}_{40} / \mathrm{Ti}$, indicando maior resistência ao fenômeno de envenenamento da superfície. Esse resultado corrobora com os resultados obtidos por voltametria cíclica, os quais também mostraram uma atividade catalítica superior desta composição para a eletro-oxidação da molécula de propanodiol.

\section{Conclusões}

Neste trabalho foram preparados eletrodos recobertos com óxidos de platina e rutênio em diferentes composições e verificada a atividade catalítica destes dispositivos durante a reação de eletro-oxidação da molécula de propanodiol. Os resultados obtidos demostraram que na faixa de potencial investigada de $300 \mathrm{mV}$ o eletrodo que apresentou maior atividade catalítica na eletro-oxidação da molécula de orgânica foi o eletrodo $\mathrm{Pt}_{60} \mathrm{Ru}_{40} / \mathrm{Ti}$, ou seja, o eletrodo contendo $40 \%$ a menos de platina. Esses resultados demostram a possibilidade de aplicação deste dispositivo em células a combustível operadas nesta faixa de potencial. 


\section{Agradecimentos}

Os autores agradecem à Fundação de Amparo à Pesquisa do Espírito Santo (FAPES) e ao Programa Institucional de Iniciação Cientifica (PIIC) da Universidade Federal do Espirito Santo.

\section{Referências}

[1] GONZALEZ, E. R. Química Nova, v.23, p.262 - 266, 2000.

[2] WATANABE, M.; MOTOO, S. Journal of Electroanalytical Chemistry and Interfacial Electrochemistry, v.60, p.267 - 273, 1975.

[3] PROFETI, L.P.R.; PROFETI, D.; OLIVI, P. International Journal of Hydrogen Energy, v.34, p.2747 - 2757, 2009.

[4] LAOUINI, E.; HAMDONI, M.; PEREIRA, M. I. S.; DOUCH, J.; MENDONÇA, M. H.; BERGHOUTE, Y.; SINGH, R. N. Journal of Applied Electrochemistry, v.38, p. 1485 - 1494, 2008.

[5] GOMES, J. F.; OLIVEIRA, V. L.; PRATTA, P. M. P.; TREMILIOSI-FILHO, G. Electrocatalysis, v.6, p. 7 - 19, 2015.

[6] SUGIYAMA, S.; TANAKA, H.; BANDO, T.; NAKAGAWA, K.; SOTOWA, K.; KATOU, Y.; MORI, T.; YASUKAWA, T.; NINOMIYA, W. Catalysis Today, v.203, p. 116 - 121, 2013.

[7] MALTANAVA, H. M.; VOROBYOVA, T. N.; VRUBLEVSKAYA, O. N. Surface \& Coatings Technology, v.254, p. 388 - 397, 2014. 